\title{
Mechanisms Mediating Bradycardia during Coronary Arteriography
}

\author{
Dwain L. Eckberg, Carl W. White, J. Michael Kioschos, and \\ Francors M. AbBoud \\ From the Cardiovascular Division, Department of Internal Medicine, Veterans \\ Administration Hospital and University of Iowa College of Medicine, \\ Iowa City, Iowa 52242
}

\begin{abstract}
A в S T R A C T Cardiac slowing occurring during diag. nostic coronary arteriography was studied in 78 patients. Comparable degrees of slowing occurred with injections into the right and into the left coronary arteries into the contralateral artery, and with injections into the coronary artery giving rise to the sinus node artery and into the contralateral artery. Rapid intracoronary injections of isosmotic dextrose solution produced significantly less slowing than comparable injections of contrast medium. Slow injections of contrast medium produced cardiac slowing comparable to that caused by rapid injections of contrast medium. However, the cardiac slowing was significantly greater than that produced by rapid injections of dextrose solution. Inhalation of $100 \%$ oxygen did not alter the heart rate response to injections of contrast medium. Atropine produced dose-related attenuation of cardiac slowing. Bradycardia persisting after cholinergic blockade was significantly greater after injections into the coronary artery supplying the sinus node than it was after injections into the contralateral artery. Coronary arteriography produced transient, occasionally profound, arterial hypotension in 38 of 41 patients in whom arterial pressures were recorded. Arterial pressure did not change in three patients.

This study suggests that the cardiac slowing which occurs during coronary arteriography in man is due primarily to a cholinergic reflex which may be a human counterpart of the Bezold-Jarisch reflex, observed heretofore only in experimental animals. This slowing appears to be mediated primarily by receptors sensitive to contrast medium, rather than by changes of coronary artery pressure, and secondarily, by direct depression of sinus node function by contrast medium.
\end{abstract}

Received for publication 17 July 1973 and in revised form 12 August 1974.

\section{INTRODUCTION}

Although bradycardia occurs commonly during diagnostic coronary arteriography (1-3), its mechanism is not understood fully, nor is there agreement upon its specific prevention (3-6). Transient hypoxia (7), direct depression of sinus node function by contrast media, ${ }^{1}$ and activation of coronary baroreflexes (4) or chemoreflexes (8) all have been proposed to explain this phenomenon. The potential seriousness of bradycardia-induced arrhythmias $(9,10)$ and the widespread use of coronary arteriography underscore the need for specific understanding of the mechanisms involved.

We have undertaken a prospective and retrospective study of the bradycardia which attends diagnostic coronary arteriography in man by measuring beat-to-beat changes of heart rate occurring during arteriography, before and after atropine, in patients with normal or diseased coronary arteries. The role of direct depression of sinus node function by contrast agents was assessed by comparing heart rate responses to injections into the coronary artery supplying the sinus node with those responses resulting from injections into the contralateral artery. The importance of contrast media to the bradycardia response was evaluated by measuring heart rate changes after comparable intracoronary injections of isosmotic dextrose solution. The role of increased intracoronary pressure in causing bradycardia was assessed by injecting contrast medium very slowly.

\section{METHODS}

78 patients averaging 48 11 (SEM) yr (range 26-67 yr) and undergoing coronary arteriography for diagnosis of

${ }^{1}$ White, C. W., D. L. Eckberg, T. Inasaka, and F. M. Abboud. 1974. Effects of angiographic contrast media on sino-atrial nodal function. Manuscript submitted for publication.

The Journal of Clinical Investigation Volume 54 December 1974.1455-1461 
chest pain were selected for analysis. Prospective studies were supplemented with cases chosen retrospectively to assure adequate numbers of patients with and without occlusive coronary artery disease. Cases were selected for analysis without knowledge of the heart rate responses to coronary arteriography, and the only ground for subsequent exclusion from the study was the inability to identify the sinus node artery on the coronary arteriogram.

Patients were studied in the postabsorptive state and were premedicated intramuscularly with $100 \mathrm{mg}$ of meperidine and $10 \mathrm{mg}$ of diazepam, given approximately $3 \mathrm{~h}$ before study, and sublingually with $0.4 \mathrm{mg}$ of nitroglycerin, given immediately before coronary arteriography. An endocardial pacing electrode was placed in the right ventricle and connected to a demand pacemaker which was left in the "off" position.

Right and left coronary ostia were selectively catheterized using either the Sones catheter introduced through a brachial arteriotomy, or a preformed catheter introduced percutaneously via the femoral artery. Meglumine diatrizoate (Renografin-76®) was injected manually in boluses of $6-8$ $\mathrm{ml}$, as forcefully as possible (injection duration about $3 \mathrm{~s}$ ) with a 10-ml syringe. The order of filming of coronary arteries was randomized. Multiple injections were made into both coronary arteries as dictated by clinical considerations.

To distinguish effects resulting from changes of coronary artery pressure from those caused by responses to angiographic dye, we compared heart rate changes after rapid, forceful injections of dye with changes resulting from slow injections (lasting about $10 \mathrm{~s}$ ) of comparable volumes of contrast medium. To evaluate the importance of contrast medium to the observed responses, heart rate changes after injections of contrast medium were compared with changes after rapid, forceful injections of $6-8 \mathrm{ml}$ of a solution of $5 \%$ dextrose in water. To demonstrate the possible role of chemoreceptors in producing heart rate changes resulting from intracoronary injection of contrast agents, we measured responses before and during inhalation of $100 \%$ oxygen. Atropine sulfate was given intravenously to demonstrate possible cholinergic mediation of heart rate changes. When feasible, both right and left coronary arteriograms were obtained before and after administration of atropine, $0.5-3.6 \mathrm{mg}$.

Successive P-P intervals were measured during held expiration, beginning before injection of contrast medium or glucose solution and continuing for 10-15 s after injection or until the maximum prolongation of the P-P interval had occurred (Fig. 1). The control P-P interval was determined by averaging measurements from 4-7 successive cardiac cycle before injection of contrast medium and was compared with the longest P-P interval after injection. Atrial rate (referred to subsequently as "heart rate") was derived from the measured P-P intervals. Only records in which normal $\mathrm{P}$-wave morphology persisted were accepted for analysis.

Cineangiograms were examined to identify the major coronary artery (left or right) giving rise to the sinus node artery. In the remainder of this manuscript, the major coronary artery (left or right) which does not give rise to the sinus node artery is referred to as the "contralateral artery." Angiograms were reviewed without prior knowledge of heart rate changes. Patients were divided into two groups: patients with normal coronary arteriograms with or without associated hemodynamic abnormalities, and patients with significant occlusive coronary artery disease. All patients included in the coronary artery disease category had greater than $75 \%$ proximal obstructive lesions of one or more major coronary arteries.

47 patients had normal coronary arteries or coronary artery lesions which were judged to be hemodynamically insignificant and 31 patients had severe coronary occlusive

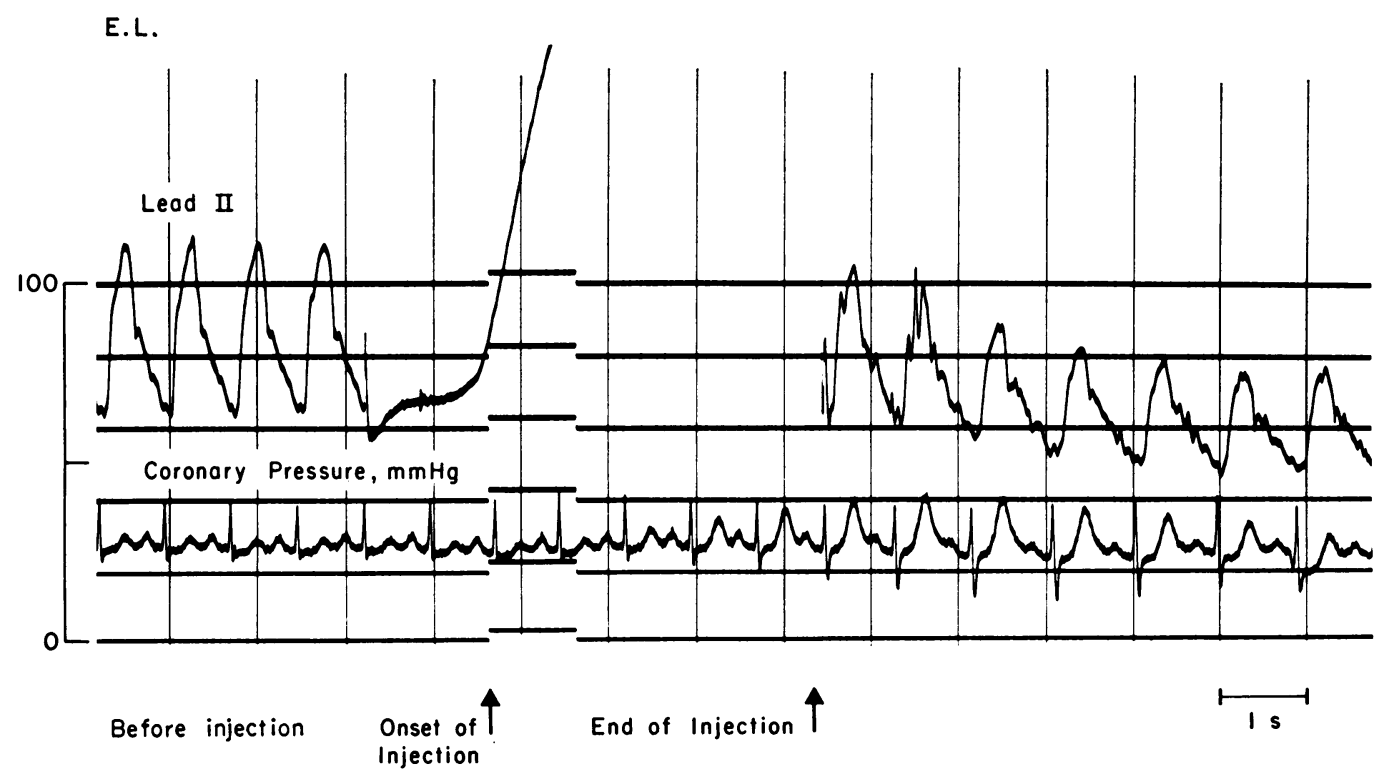

FIGURE 1 Continuous record obtained during coronary arteriogram showing coronary artery pressure obtained through the injection catheter and lead II of the electrocardiogram. In this instance, average heart rate (calculated from P-P intervals) before injection of contrast medium was 79 and fell to a minimum of 63 beats/min after injection. Calculated mean arterial pressure fell from 81 to $55 \mathrm{~mm} \mathrm{Hg}$. 
disease. In eight, disease was limited to one coronary artery; in four of these patients, the sinus node artery arose from the diseased vessel. In 23 patients, there was severe, diffuse disease involving both major coronary arteries. Three patients with coronary artery disease treated with aortocoronary saphenous vein bypass grafts were studied by injecting contrast medium selectively into the proximal, aortic anastomoses. The sinus node artery arose from the right coronary artery in 43 of the 75 patients, or $57 \%$.

Eight patients were taking digoxin at the time of study. 13 patients were being treated with propranolol, which was discontinued about $12 \mathrm{~h}$ before catheterization. Arterial pressure averaged $120 / 70 \mathrm{~mm} \mathrm{Hg}$; nine patients had systolic pressures above $140 \mathrm{~mm} \mathrm{Hg}$, but none had diastolic pressures above $90 \mathrm{~mm} \mathrm{Hg}$. Age, control heart rate, arterial pressure, and the number of patients taking digoxin or propranolol were comparable in the two groups studied.

Statistical significance was determined using paired and unpaired $t$ tests and the chi-square test, and regression analyses were performed using the least-squares method (11).

\section{RESULTS}

Heart rate changes with coronary arteriograms. Typical electrocardiographic and arterial pressure changes occurring during diagnostic coronary arteriography are shown in Fig. 1. Intracoronary injections of contrast medium led to cardiac slowing in all patients. Heart rate changes during coronary arteriography were reproducible: the average cardiac slowing produced by the first injection of contrast medium was $22.2 \pm 2.7$ and that caused by the second injection into the same coronary artery was $24.2 \pm 3.2$ beats $/ \mathrm{min}$ (Fig. 2). Regression analysis of these data yielded a correlation coefficient of 0.82 and a slope of 0.998 .

Heart rate data for all patients before atropine are summarized in Table I. The average maximum cardiac slowing of $24.3 \pm 2.7$ beats $/ \mathrm{min}$ resulting from injec-

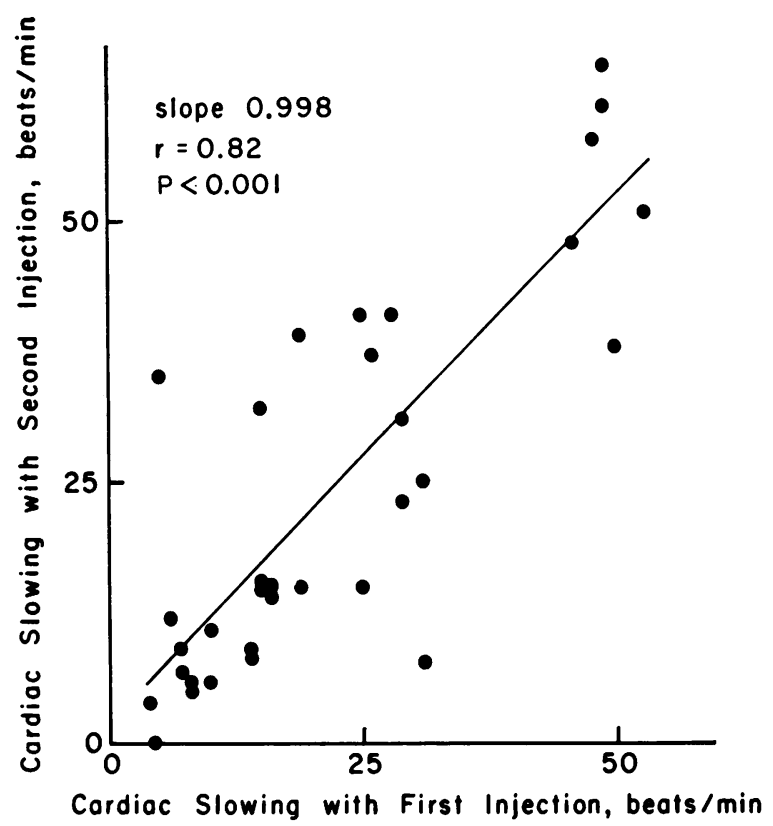

FIGURE 2 Regression analysis of the heart rate slowing produced by the first intracoronary injection of contrast medium and the second.

tions into the right coronary artery was not significantly different from the average maximum of 26.5 \pm 2.8 which followed injections into the left coronary artery. Similarly, slowing with injections into the coronary artery supplying the sinus node averaged 25.1 \pm 3.0 beats $/ \mathrm{min}$ and was $25.9 \pm 2.6$ with injections into the contralateral coronary artery. In three patients, injections of contrast medium into the aortic ostia of saphenous vein grafts perfusing the midright coronary artery produced cardiac slowing averaging 35 (range 19-50) beats/min.

TABLE I

Data from Patients Before Atropine

\begin{tabular}{|c|c|c|c|c|c|c|}
\hline & \multirow[b]{2}{*}{ Age } & \multirow[b]{2}{*}{$\begin{array}{l}\text { Arterial } \\
\text { pressure }\end{array}$} & \multicolumn{2}{|c|}{ Sinus node artery } & \multicolumn{2}{|c|}{ Contralateral } \\
\hline & & & $\begin{array}{l}\text { Control } \\
\text { HR }\end{array}$ & $\Delta \mathrm{HR}$ & $\begin{array}{l}\text { Control } \\
\text { HR }\end{array}$ & $\Delta \mathrm{HR}$ \\
\hline & $y r$ & $m m H g$ & \multicolumn{2}{|c|}{ beats $/ \min$} & \multicolumn{2}{|c|}{ beats/min } \\
\hline $\begin{array}{l}\text { I. Normal coronary arteries } \\
n\end{array}$ & $46 \pm 2$ & $122 / 71$ & $\begin{array}{c}83 \pm 3 \\
24\end{array}$ & $-23.5 \pm 3.5$ & $\begin{array}{c}81 \pm 3 \\
25\end{array}$ & $-22.0 \pm 3.1$ \\
\hline $\begin{array}{l}\text { II. Occlusive coronary disease } \\
n\end{array}$ & $51 \pm 2$ & $118 / 70$ & $\begin{array}{c}83 \pm 3 \\
11\end{array}$ & $-27.8 \pm 5.1$ & $\begin{array}{c}84 \pm 3 \\
15\end{array}$ & $-32.3 \pm 4.3$ \\
\hline $\begin{array}{l}\text { III. All patients } \\
n\end{array}$ & $48 \pm 1$ & $120 / 70$ & $\begin{array}{c}83 \pm 3 \\
35\end{array}$ & $-25.1 \pm 3.0$ & $\begin{array}{c}82 \pm 2 \\
40\end{array}$ & $-25.9 \pm 2.6$ \\
\hline
\end{tabular}

Mean values $\pm \mathrm{SE}$ for all patients. $\mathrm{HR}$, heart rate; $\boldsymbol{n}$ number of patients.

There were no significant differences between values obtained from patients with normal coronary arteries and those obtained from patients with coronary occlusive disease. 
TABLE $^{-}$II

Heart_Rate Responses to Rapid and Slow Intracoronary Artery Injections

\begin{tabular}{|c|c|c|c|c|c|c|}
\hline \multirow[b]{2}{*}{ Patient } & \multicolumn{2}{|c|}{ (a) Rapid dye injection } & \multicolumn{2}{|c|}{ (b) Slow dye injection } & \multicolumn{2}{|c|}{ (c) Rapid dextrose injection } \\
\hline & $\begin{array}{c}\text { Control } \\
\text { HR }\end{array}$ & $\Delta \mathrm{HR}$ & $\begin{array}{c}\text { Control } \\
\text { HR }\end{array}$ & $\Delta \mathrm{HR}$ & $\begin{array}{c}\text { Control } \\
\text { HR }\end{array}$ & $\Delta \mathrm{HR}$ \\
\hline & \multicolumn{2}{|c|}{ beats/min } & \multicolumn{2}{|c|}{ beats $/ \min$} & \multicolumn{2}{|c|}{ beats/min } \\
\hline H. H. & 83 & -18 & 88 & -5 & 88 & -5 \\
\hline D. B. & 60 & -30 & 58 & -2 & 60 & 0 \\
\hline E. L. & 83 & -8 & 83 & -28 & 83 & -8 \\
\hline H. D. & 83 & -26 & 88 & -9 & 88 & -13 \\
\hline M. C. & 88 & -43 & 94 & -29 & 88 & -5 \\
\hline N. P. & 94 & -40 & 94 & -34 & 94 & -22 \\
\hline A. P. & 79 & -19 & & & 79 & -4 \\
\hline L. D. & 88 & -13 & & & 94 & -6 \\
\hline K. M. & 79 & -27 & & & 83 & -8 \\
\hline L. M. & 58 & -35 & & & 54 & 0 \\
\hline E. P. & 63 & -21 & & & 60 & -4 \\
\hline S. M. & 71 & -3 & & & 71 & -3 \\
\hline Mean \pm SEM & $77.4 \pm 3.4$ & $-23.6 \pm 3.6$ & $84.2 \pm 5.5$ & $-17.8 \pm 5.7$ & $78.5 \pm 4.0$ & $-6.5 \pm 1.7$ \\
\hline
\end{tabular}

Nonpaired $t$ test of $\Delta \mathrm{HR}: a$ vs. $b, \mathrm{NS} ; a$ vs. $c P<0.001 ; b$ vs. $c, P<0.05$.

Maximum cardiac slowing during (left or right) coronary angiography was greater in patients with occlusive coronary artery lesions than in those without significant coronary disease $(35.1 \pm 4.2$ vs. $27.8 \pm 3.3$ beats $/ \mathrm{min})$, but the difference was not significant $(P$ $>0.05$ ). The heart rate responses of a subgroup of 12 patients with normal coronary arteriograms, but abnormal hemodynamic findings were comparable to those of patients with entirely normal catheterization data. There were no significant differences between the responses of patients who had taken digoxin or pro-

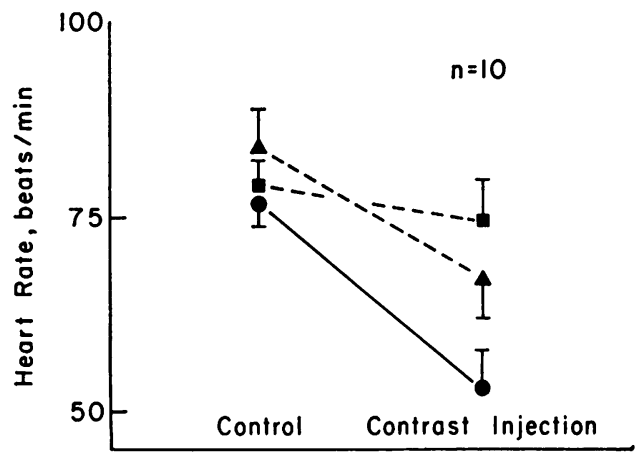

FIgURE 3 Average heart rates before and after injections of contrast medium and physiologic glucose solution, injected forcefully, or with minimum force. Brackets encompass 1 SEM. Responses to rapid and slow injections of contrast medium were comparable $(P>0.20)$ but were significantly greater than responses to isosmotic glucose solution $(P<0.005$ and $P<0.05$, respectively). Heart rate changes caused by: glucose under pressure ( $\square---\square)$, contrast under pressure ( $\bullet)$, and contrast without pressure $(\Delta--\Delta)$. pranolol and those of patients not on medical therapy.

The duration of the chronotropic effects of coronary arteriography was determined in 11 patients by plotting instantaneous heart rate from the onset of injection until control rates were reestablished. The average duration of bradycardia was comparable $(P$ $>0.5$ ) with injections into the left and right coronary arteries $(25.9 \pm 5.6$ vs. $20.5 \pm 5.4 \mathrm{~s})$ and with injections into the coronary artery supplying the sinus node and the contralateral coronary artery $(24.5 \pm 3.4$ vs. 25.2 $\pm 3.8 \mathrm{~s}$ ).

Heart rate changes resulting from rapid and slow intracoronary injections of angiographic dye and rapid intracoronary injections of isosmotic dextrose solution are shown in Table II. Rapid injections of contrast medium led to greater cardiac slowing than slow injections of contrast medium $(23.6 \pm 3.6$ vs. $17.8 \pm 5.7$ beats $/ \mathrm{min}$ ), but the difference was not significant $(P$ $>0.20$ ). Rapid injections of physiologic dextrose solution into either coronary artery led to minor cardiac slowing (averaging $6.5 \pm 1.7$ beats $/ \mathrm{min}$ ) which was significantly less than slowing observed after rapid $(P<0.005)$ or slow $(P<0.05)$ injections of contrast medium (Fig. 3). Slowing with rapid injections of dextrose solution exceeded 10 beats/min in only two patients. In both instances, these injections were made into the coronary artery giving rise to the sinus node artery, raising the possibility that slowing was mediated by sudden changes of sinus node artery pressure $(12,13)$.

In six patients, inhalation of $100 \%$ oxygen raised the average $\mathrm{Po}$, from $70 \pm 8$ to $349 \pm 45 \mathrm{~mm} \mathrm{Hg}$, but

1458 D. L. Eckberg, C. W. White, J. M. Kioschos, and F. M. Abboud 


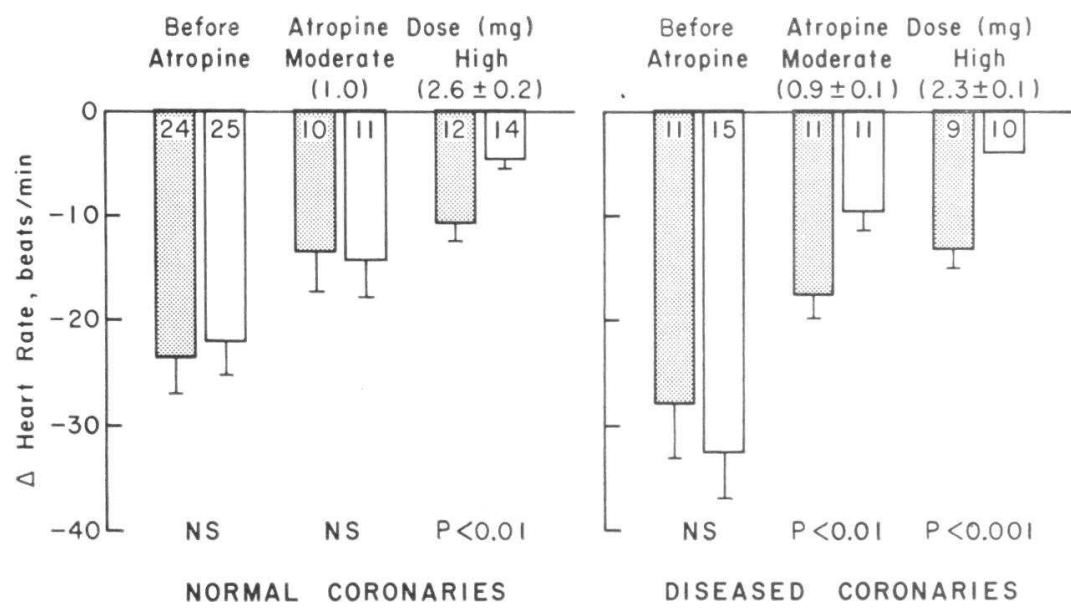

FIGURE 4 Influence of cholinergic blockade upon heart rate responses to coronary arteriography. Stippled bars represent responses to injections into the coronary artery supplying the sinus node and clear bars represent responses to injections into the contralateral coronary artery. Brackets encompass 1 SEM. The average cardiac slowing with injections into the contralateral coronary artery was significantly $(P<0.025)$ lower in patients (with or without coronary artery disease) receiving high than those receiving moderate doses of atropine. Responses to injections into the coronary artery supplying the sinus node were not significantly different in moderate and high dose groups.

did not alter significantly the control heart rate $(81$ \pm 5 vs. $79 \pm 5$ beats $/ \mathrm{min}$ ) or the maximum cardiac slowing $(25.5 \pm 4.8$ vs. $26.8 \pm 3.6$ beats $/ \mathrm{min})$ resulting from the intracoronary injection of contrast medium.

Heart rate changes with angiography after atropine. 0.5 to $3.6 \mathrm{mg}$ of atropine was given to 26 patients with normal coronary arteries and to 23 patients with coronary artery disease. There were no significant differences between the average doses given to the groups of patients with and without coronary artery disease. Patients in each group were subdivided for statistical analysis into those receiving "moderate" doses of atropine $(0.5-1.5 \mathrm{mg})$ or "high" doses $(2.0-3.6 \mathrm{mg})$. The majority $(84 \%)$ of patients in the moderate dose group received $1.0 \mathrm{mg}$ atropine and only two patients received doses between 1.0 and $1.5 \mathrm{mg}$. The variability of atropine dose in this group resulted from the retrospective addition of cases for analysis (see Methods). All patients included in the high dose category were studied prospectively and received either $2.0 \mathrm{mg}$ or 0.04 $\mathrm{mg} / \mathrm{kg}$ atropine. High doses of atropine were tolerated well and did not lead to ventricular irritability (14).

In both groups of patients, there was an inverse relationship between atropine dosage and the magnitude of cardiac slowing caused by contrast medium injections into the coronary artery not supplying the sinus node (Fig. 4). In patients with normal coronary arteries, slowing averaged $14.2 \pm 3.4$ beats $/ \mathrm{min}$ in those given moderate doses of atropine and $4.6 \pm 0.8$ in patients given high doses $(P<0.05)$. Comparable attenuation by atropine occurred in patients with coronary artery disease $(9.5 \pm 1.8$ vs. $3.9 \pm 1.2$ beats $/ \mathrm{min}, P<0.025)$. In contrast, bradycardia caused by injections into the coronary artery supplying the sinus node was not influenced significantly by atropine dosage (Fig. 4) in either group.

With high dose atropine, significantly greater cardiac slowing occurred with injections into the artery supplying the sinus node than into the contralateral coronary artery in patients with $(P<0.001)$ and without $(P<0.01)$ coronary artery disease. With moderate dose atropine, a significant $(P<0.01)$ difference was observed only in patients with coronary artery disease. Although bradycardia caused by injections into the coronary artery supplying the sinus node before and after atropine tended to be greater in patients with coronary artery disease than in patients with normal coronary arteries, the differences were not significant.

Arterial pressure responses to coronary angiography. Arterial pressures were recorded before and after coronary arteriography in 41 patients. Mean arterial pressure during injections fell in 38 patients, by an average of $18.4 \pm 1.9 \mathrm{~mm} \mathrm{Hg}$. Arterial pressure did not change in three patients. The reduction of arterial pressure was comparable $(P>0.20)$ in patients with and without coronary artery disease. After atropine, the fall of arterial pressures was significantly $(P<0.025)$ less than before and averaged $10.5 \pm 1.7 \mathrm{~mm} \mathrm{Hg}$.

\section{DISCUSSION}

Intracoronary injections of angiographic contrast agents lead to reproducible cardiac slowing and re- 
duction of arterial pressure. Our data suggest that cardiac slowing results primarily from a cholinergic reflex triggered by the presence of contrast medium within the coronary arterial tree, which appears to be a human counterpart of the experimentally observed Bezold-Jarisch reflex. In the present study, we attempted to define the location of receptors mediating this response, to characterize their function, and to demonstrate possible reflex activation.

Our findings suggest that receptors are present in the distribution of both major coronary arteries since comparable slowing occurred with injections into the right and into the left coronary ostia. These conclusions differ from those of Takaro, Dart, Scott, Fish, and Nelson (6), Baltaxe, Amplatz, and Levin (15), and Weidner, MacAlpin, Hanafee, and Kattus (16) who reported that heart rate changes are greater with injections into the right coronary artery than into the left.

Our studies demonstrate further that sinus node receptors are not primarily responsible for the bradycardia since comparable slowing occurred with injections into the coronary artery supplying the sinus node and into the contralateral artery. Studies in dogs ${ }^{1}$ demonstrate that sinus node function is directly depressed by hyperosmolar contrast media, an effect which is not altered by cholinergic blockade. The results of the present investigation in man support these observations, but direct sinus node depression was not demonstrable until after adequate cholinergic blockade. After blockade of the reflex component, however, significantly greater slowing resulted from injections into the coronary artery supplying the sinus node than into the contralateral coronary artery. Thus, although our results demonstrate that direct sinus node depression occurs during coronary arteriography, the contribution is masked by a stronger, reflex component mediated by other receptors. In three patients, slowing resulted from injections of contrast medium into the midright coronary artery via aortocoronary bypass grafts, suggesting that these receptors are not restricted to the distribution of the proximal coronary arterial tree.

Receptors which may contribute to reflex cardiac slowing during coronary arteriography include coronary baroreceptors and coronary, myocardial, or aortic chemoreceptors. Recent evidence has favored participation of coronary baroreceptors in mediation of this response. Brown (17) has shown that sudden increases of intracoronary artery pressure increase afferent nerve traffic from the heart. Carson and Lazzara (4) produced bradycardia by injecting contrast medium selectively into the coronary ostia of dogs and attributed this heart rate change to activation of coronary artery baroreflexes. Studies in dogs from our laboratory ${ }^{1}$ have shown that rapid injections of contrast agents or isosmotic saline through catheters placed in the coronary ostia, consistently produce small, transient elevations of intracoronary artery pressure which might be expected to trigger coronary artery baroreflexes.

Our study suggests, however, that heart rate changes seen during coronary arteriography in man are not mediated primarily by changes of intracoronary artery pressure. Rapid, forceful injections of contrast medium produced much greater cardiac slowing than comparable injections of dextrose solution. In addition, very slow injections of contrast medium (slow injections into the coronary ostia of the dog do not elevate coronary artery pressure ${ }^{1}$ also led to significantly greater slowing than rapid injections of dextrose solution. Rapid injections of contrast medium produced slightly more cardiac slowing than slow injections but the difference was not statistically significant. These results suggest that angiographic dye itself may be more important in producing cardiac slowing than changes of coronary artery pressure incident to injection.

We considered the possibility that the effects of contrast medium might be mediated by "coronary chemoreceptors" (18), activated by changes in their natural chemical environment (reduced arterial $\mathrm{Poz}_{2}$ or oxygen content) or by contrast medium. Coronary arteriograms would not be expected to lower $\mathrm{Po}_{2}$ because the $\mathrm{Po}_{2}$ of contrast medium exposed to atmosphere is similar to that of arterial blood. Moreover, significant bradycardia occurred when arteriograms were performed during inhalation of $100 \%$ oxygen, when systemic, and presumably coronary arterial $\mathrm{Pos}_{3}$ were elevated. Rapid injections of dextrose solution would be expected to lower coronary arterial oxygen content as much as rapid injections of contrast medium, but the latter led to significantly greater cardiac slowing. In addition, slow injections of the same volume of contrast medium, which should have lowered coronary arterial oxygen content less than rapid injections, led to comparable bradycardia.

The minor slowing caused by intracoronary injections of isosmotic dextrose in the present study is not explained fully. In the open-chest dog, rapid injections of saline into the ostium of the right coronary artery led to minor cardiac slowing which was prevented by prior occlusion of the sinus node artery (which arose from the right coronary artery in each instance). In the present study, rapid injections of dextrose solution into the coronary arteries led to cardiac slowing exceeding 10 beats $/ \mathrm{min}$ in only two patients. In both instances, injections were made into the coronary artery giving rise to the sinus node artery. In the dog, isosmotic glucose solution does not directly influence sinus 
node function, but James and Nadeau (12) and Hashimoto, Tanaka, Hirata, and Chiba (13) have shown that sudden elevations of pressure within the sinus node artery lead to bradycardia. Our study raises the possibility that the minor cardiac slowing which results from coronary injections of glucose solution in man, may be mediated in part by increased pressure in the sinus node artery.

Our studies support the conclusions of others (4, $19,20)$ that cardiac slowing occurring during coronary arterial injection of contrast agents is mediated primarily by a cholinergic reflex. Significant slowing was observed when contrast medium was injected into coronary arteries not supplying the sinus node and this supports the existence of a reflex arc. Further evidence is found in the response to atropine: cholinergic blockade produced a significant, dose-related attentuation of the cardiac slowing caused by intracoronary injection of contrast medium.

The present study documents a fall of systemic arterial pressure with coronary arteriography, and other studies from this laboratory (21) have shown that coronary arteriography leads to a reduction of cardiac output and forearm blood flow. In these studies, the fall of arterial pressure and the reduction of forearm blood flow were attenuated significantly by atropine. These findings suggest that contrast agents may function as "detector substances" (22) in triggering a cholinergic depressor reflex in man which is comparable to the Bezold-Jarisch reflex reported heretofore in experimental animals.

\section{ACKNOWLEDGMENTS}

This work was supported by NIH grants HL 14388 and HL 05729, and by grants from the Veterans Administration and the Iowa Heart Association.

\section{REFERENCES}

1. Benchimol, A., and E. M. McNally. 1966. Hemodynamic and electrocardiographic effects of selective coronary angiography in man. N. Engl. J. Med. 274: 1217-1224.

2. MacAlpin, R. N., W. A. Weidner, A. A. Kattus, Jr., and W. N. Hanafee. 1966. Electrocardiographic changes during selective coronary cineangiography. Circulation. 34: 627-637.

3. Coskey, R. L., and O. Magidson. 1967. Electrocardiographic response to selective coronary arteriography. Br. Heart J. 29 : 512-519.

4. Carson, R. P., and R. Lazzara. 1970. Hemodynamic responses initiated by coronary stretch receptors with special reference to coronary arteriography. Am. J. Cardiol. 25: 571-578.

5. Benchimol, A., and E. M. McNally. 1967. Atrial pacing during selective coronary angiography. Br. Heart $J$. 29 : 767-769.

6. Takaro, T., C. H. Dart, Jr., S. M. Scott, R. G. Fish, and W. M. Nelson. 1968. Coronary arteriography. Ann. Thorac. Surg. 5: 213-221.

7. Gootman, N., A. M. Rudolph, and N. M. Buckley. 1970. Effects of angiographic contrast media on cardiac function. Am. J. Cardiol. 25 : 59-65.

8. Zelis, R., D. T. Mason, J. F. Spann, Jr., and E. A Amsterdam. 1970. Stimulation of myocardial chemoreceptors in man: forearm arteriolar dilatation induced by contrast media during coronary arteriography. Circulation. 42 (Suppl. III) : III-42. (Abstr.)

9. Adgey, A. A. J., J. S. Geddes, H. C. Mulholland, D. A. J. Keegan, and J. F. Pantridge. 1968. Incidence, significance, and management of early bradyarrhythmia complicating acute myocardial infarction. Lancet. 2: 10971101.

10. Han, J., D. Millet, B. Chizzonitti, and G. K. Moe. 1966. Temporal dispersion of recovery of excitability in atrium and ventricle as a function of heart rate. $\mathrm{Am}$. Heart J. 71 : 481-487.

11. Snedecor, G. W., and W. G. Cochran. 1967. Statistical Methods. Iowa State University Press, Ames. 6th edition.

12. James, T. N., and R. A. Nadeau. 1963. Sinus bradycardia during injections directly into the sinus node artery. Am. J. Physiol. 204: 9-15.

13. Hashimoto, K., S. Tanaka, M. Hirata, and S. Chiba. 1967. Responses of the sino-atrial node to change in pressure in the sinus node artery. Circ. Res. 21: 297304.

14. Massumi, R. A., D. T. Mason, E. A. Amsterdam, A. DeMaria, R. R. Miller, M. M. Scheinman, and R. Zelis. 1972. Ventricular fibrillation and tachycardia after intravenous atropine for treatment of bradycardias. $N$. Engl. J. Med. 287 : 336-338.

15. Baltaxe, H. A., K. Amplatz, and D. C. Levin. 1973. Coronary Angiography. Charles C. Thomas, Publishers, Springfield, Ill. 138.

16. Weidner, W., R. MacAlpin, W. Hanafee, and A. Kattus. 1965. Percutaneous transaxillary selective coronary angiography. Radiology. $85: 652-657$.

17. Brown, A. M. Mechanoreceptors in or near the coronary arteries. 1965. J. Physiol. (Lond.). 177: 203-214.

18. Dawes, G. S., and J. H. Comroe, Jr. 1954. Chemoreflexes from the heart and lungs. Physiol. Rev. 34: 167201.

19. Smith, R. F., J. W. Harthorne, and C. A. Sanders. 1967. Vector-cardiographic changes during intracoronary injections. Circulation. 36: 63-76.

20. Glassman, E., and G. Roberts. 1969. Prevention of arrhythmias during coronary arteriography by prophylactic atropine. Circulation. 40(Suppl. III): III-90. (Abstr.)

21. White, C. W., D. L. Eckberg, J. M. Kioschos, and F. M. Abboud. 1973. A study of coronary artery reflexes in man. Circulation. 48:(Suppl. IV): IV-65. (Abstr.)

22. Krayer, O. 1961. The history of the Bezold-Jarisch effect. Arch. Exp. Pathol. Pharmacol. 240: 361-368. 